\title{
Neck of Femur Fractures within Two Hours of a Meal
}

Nair $\mathbf{S}^{1,3}$, Piscitelli $\mathbf{D}{ }^{2,3}$, Tam $\mathbf{K}^{1,3}$, Nair $\mathbf{P S}^{1,3}$ and Visvanathan $\mathbf{R}^{1,3}$

${ }^{1}$ Aged and Extended Care Services, The Queen Elizabeth Hospital, Central Adelaide Local Health Network, Adelaide, South Australia

${ }^{2}$ School of Health Sciences, University of South Australia, Adelaide, South Australia

${ }^{3}$ Adelaide Geriatrics Training and Research with Aged Care (G-TRAC) Centre, School of Medicine, University of Adelaide, Adelaide, South Australia

*Corresponding author: Shailaja Nair, The Queen Elizabeth Hospital, 28 Woodville Road, Woodville South, South Australia 5011, Australia, Tel: +61 8 82226000; Fax: +61 8 82228593; E-mail: Nair.Shailaja@health.sa.gov.au

Rec date: Oct 07, 2014; Acc date: Dec 27, 2014; Pub date: Dec 29, 2014

Copyright: @ 2014 Nair S, et al. This is an open-access article distributed under the terms of the Creative Commons Attribution License, which permits unrestricted use, distribution, and reproduction in any medium, provided the original author and source are credited.

Abstract
Aim: To determine in older hospitalized patients the prevalence of neck of femur (NOF) fractures occurring within
two hours of a meal and the factors associated with these fractures.
Methods: A prospective study was undertaken within the orthopedic unit of a 320 bed general hospital.
Consecutive first admissions of patients aged 70 years and over with fragility NOF fractures between March and
November 2011 were included. Patients or their careers were interviewed using a standardised questionnaire.
Results: 120 patients were recruited. The median age was 84.0 years. Falls within two hours of a meal occurred
in $20.8 \%$ of patients. Independent factors associated with falls occurring within two hours of a meal were symptoms
of hypotension prior to a fall (OR 5.2 , Cl $1.342-20.129 ; \mathrm{P}=0.017$ ) and being in residential care (OR $3.0, \mathrm{Cl}$
$1.009-8.687$; $\mathrm{P}=0.048$ ).
Conclusion: Patients who sustained a NOF fracture within two hours of a meal were more likely to be from
residential care and experience symptoms associated with hypotension before the fall compared with patients who
experienced a fall after two hours of a meal; identification of the reasons for hypotension and potential management
strategies require further investigation.

Keywords: Aging; Blood pressure; Fractures; Hypotension; Postprandial

\section{Introduction}

Falls occur frequently in older people, with a prevalence of $30 \%$ among community dwelling individuals and $50 \%$ among older people living in residential care facilities [1]. A major consequence of falls is fractures which pose a considerable public health issue as they are associated with detrimental physical and psychological morbidity $[2,3]$ as well significant health care costs [4]. Given that there has been a worldwide increase in the total number of fractures secondary to falls over the past 50 years which is projected to continue [2], falls appear likely to pose a future challenge which is crucial to address. The implications for hip fractures which occur among a third of all fractures is particularly dismal as reflected by a mortality of $10 \%-28 \%$ at six months [5], 33\% mortality in the first year, permanent functional disabilities in $32 \%-80 \%[6,7]$ and institutionalization in up to $35 \%$ of individuals [8]. Compared to other fractures, medical costs as a result of hip fractures are the highest with estimates of costing $\$ 5$ billion dollars annually [9]. Therefore, where a hip fracture can be prevented, it is important to do so.

Given that $90 \%$ of hip fractures in older people are a consequence of falls [10], a key component of hip fracture prevention strategies requires the identification of risk factors associated with falls. The risk factors for falls in older people are typically multifactorial and the falls risk increases from $8 \%$ among those with no risk factors to $78 \%$ among those with four or more risk factors [11]. Both orthostatic [12,13] and postprandial hypotension $(\mathrm{PPH})$ have been suggested to be possible risk factors for falls $[11,14-16]$; the latter is defined commonly as a 20 $\mathrm{mmHg}$ reduction in systolic blood pressure (BP) [17] or a decline in systolic BP to less than $90 \mathrm{mmHg}$ from a pre-ingestion pressure of greater than $100 \mathrm{mmHg}$ within two hours of a meal [18].

Although the fracture prevalence in older people according to the time of day has been previously published $[19,20]$, there have been no studies reporting the prevalence of neck of femur (NOF) fractures as a result of a fall occurring within two hours of a meal. Therefore, the aim of this study was to determine in older hospitalized patients the prevalence of NOF fractures occurring within two hours of a meal and the factors associated with these fractures.

\section{Materials and Methods}

\section{Participants}

A prospective study of all overnight emergency patients aged 70 years and over, admitted to the orthopedic unit of a 320 bed general teaching hospital in South Australia with NOF fractures following a low impact fall, was undertaken between March and November 2011. In this study, a low impact fall was defined as an unexpected event in which an older individual falls to the ground from an upper level or the same level as the individual. Approval for this study was provided by the Central Northern Adelaide Health Service Ethics for Human Research Committee (approval number: 2010178). 
Page 2 of 5

\section{Protocol}

Patients or their carers were interviewed using a standardized questionnaire consisting of questions relating to demographic variables and the circumstances of the patient's fall within 72 hours of admission. The time of the fracture in relation to meal ingestion was classified as either within two hours of a meal or longer. The time periods of the falls were categorised as $0000-0600 \mathrm{~h}, 0600-1200 \mathrm{~h}$, 1200-1800 h, 1800-2400 $\mathrm{h}$ with the latter three time periods considered to correspond to breakfast, lunch and dinner, respectively. In this study, a change in posture preceding a fall was considered relevant if the patient or carer reported that the patient had transferred from either a supine to sitting or standing position or from a sitting to standing position. Patients were considered symptomatic of PPH before the fall if the following symptoms were reported: giddiness, light-headedness, syncope or blurred vision [21]. Global assessment of health status was assessed by calculating the Charlson Comorbidity Index (CCI), a measure of comorbidity, which predicts the one-year mortality rate for a patient by taking into account both the number and severity of 19 pre-defined comorbid conditions [22]. Each condition is assigned a score of $1,2,3$ or 6 depending on the mortality risk associated with the condition. The scores are summed and a total score is derived. The one-year mortality rates for the different scores are: ' 0 ': $12 \%$; ' $1-2$ ': $26 \%$; ' $3-4$ ': $52 \%$; and 'greater than or equal to 5 ': $85 \%$ [22]. Premorbid functional status was assessed using the Katz Index based on proxy reports and referenced to two weeks prior to admission [23]. The Katz Index assesses an individual's independence in performing six basic-care skills (feeding, bathing, grooming, dressing, using the toilet and transferring between bed and chair). Each activity is scored ' 1 ' if independent and ' 0 ' if dependent in performing these tasks and the scores are summed. A score of 6 indicates full function, 4 indicates moderate impairment, and 2 or less indicates severe functional impairment [24]. Cognition was assessed by history of confusion and the Mini Mental State Examination (MMSE) [23]. The MMSE is a widely used screening instrument for measuring global cognitive status. The score is obtained from an aggregate of patient performance on questions around orientation, memory, concentration, language, praxis, and visuoconstructional skills. Scores range from 0 to 30 . Cognitive impairment is defined as either the mention of confusion in the medical records, a formal diagnosis of delirium, dementia or a MMSE of 24 or less.

\section{Statistical analysis}

Patients' characteristics were summarized using means and standard deviations, or frequencies and percentages, as appropriate. Comparisons between patient groups were performed using the chisquared test and independent-samples t-test. One-sample chi-square test was used to determine if falls occurred randomly across the different time categories. Logistic regression analysis was performed to determine the independent associations of variables with fractures occurring within two hours of a meal. Variables associated with the outcome with a $P$ value less than 0.05 were entered into a multivariable logistic regression model and odds ratios with corresponding 95\% confidence intervals were derived. The final model contained three independent variables (pre-admission residence, presence of symptoms, history of recurrent falls). All statistics were performed with the assistance of a professional biostatistician using SPSS statistical software, version 20 (SPSS Inc., Chicago, Illinois, USA).

\section{Results}

There were a total of 138 admissions eligible for this study over the nine month study period with 120 patients included in this study. Data from the remaining 18 patients were missing due to a shorter hospital admission duration or admission during holiday periods. There were no statistically significant differences between the included and the missed patients for age (included: $84.2+7.5$ years vs. missed: $85.7+8.1$ years; $\mathrm{P}=0.452$ ), gender (included: male $26.7 \%$ vs. missed: male $27.7 \%$; $\mathrm{P}=0.921$ ) and percentage admitted from their own home (included: $64.2 \%$ vs. missed: $66.6 \% ; \mathrm{P}=0.836$ ).

Table 1 summarizes the patients' characteristics and compares the overall group with those who fell within and after two hours of a meal. Generally, the patients in this study were considered very old with a mean age of $84.2(+7.5)$ years. In keeping with this age demographic, $73.3 \%$ of patients were female, $64.2 \%$ of patients were living at home prior to hospitalization while the remaining $35.8 \%$ of patients were from residential care. $32.5 \%$ of all falls were related to a change in posture. The possibility of cognitive impairment was noted in $57.5 \%$ of patients. In keeping with this, only $56.7 \%$ of patients provided a falls history themselves while collaborative history was obtained from carers for $43.4 \%$ of patients.

\begin{tabular}{|c|c|c|c|c|}
\hline Variables & Total Population $n=120$ & $\begin{array}{l}\text { Fractures occurring }<2 \\
\text { hours after a meal } n=25\end{array}$ & $\begin{array}{l}\text { Fracture occurring }>2 \text { hours } \\
\text { after a meal } n=95\end{array}$ & $P$ value \\
\hline Age, years (Mean/SD) & $84.2(7.5)$ & $83.3(5.5)$ & $84.4(7.9)$ & 0.384 \\
\hline \multicolumn{5}{|l|}{ Gender, n (\%) } \\
\hline Male & $32(26.7)$ & $4(16)$ & $28(29.5)$ & 0.212 \\
\hline Female & $88(73.3)$ & $21(84)$ & $67(70.5)$ & \\
\hline \multicolumn{5}{|c|}{ Preadmission residence, $\mathrm{n}(\%)$} \\
\hline Home & $77(64.2)$ & $10(40)$ & $67(70.5)$ & $0.005^{*}$ \\
\hline Residential care & $43(35.8)$ & $15(60)$ & 28 (29.5) & \\
\hline \multicolumn{5}{|l|}{ Time of fracture, $n(\%)$} \\
\hline 0600-1200 & $41(34.2)$ & $11(44)$ & $30(31.6)$ & $0.224^{\wedge}$ \\
\hline $1200-1800$ & $36(30)$ & $8(32)$ & $28(29.5)$ & \\
\hline
\end{tabular}




\begin{tabular}{|c|c|c|c|c|}
\hline $1800-2400$ & $30(25)$ & $6(24)$ & $24(25.2)$ & \\
\hline $0000-0600$ & $13(10.8)$ & $0(0)$ & $13(13.7)$ & \\
\hline \multicolumn{5}{|l|}{ Source of history, $n(\%)$} \\
\hline Patient & $68(56.7)$ & $15(60)$ & $53(55.8)$ & 0.705 \\
\hline Carer & $52(43.3)$ & $10(40)$ & $42(44.2)$ & \\
\hline Change in posture preceding fall, $\mathrm{n}(\%)$ & $39(32.5)$ & $9(36)$ & $30(31.6)$ & 0.903 \\
\hline $\begin{array}{l}\text { Hypotensive symptoms prior to fall (giddiness, light- } \\
\text { headedness, syncope or blurred vision), } \mathrm{n}(\%)\end{array}$ & $12(10.0)$ & $6(24)$ & $6(6.3)$ & $0.032^{*}$ \\
\hline Number of medications (Mean /SD) & $5.8(3.2)$ & $6.3(3.6)$ & $5.6(3.1)$ & 0.35 \\
\hline$>1$ fall in preceding 12 months, $n(\%)$ & $65(54.2)$ & $18(72)$ & $47(49.5)$ & $0.044^{*}$ \\
\hline $\mathrm{CCl},($ Mean/SD) & $2.4(1.9)$ & $2.2(2.0)$ & $2.4(1.9)$ & 0.648 \\
\hline Heart Disease, $n(\%)$ & $64(53.3)$ & 11 & 53 & 0.293 \\
\hline Hypertension, n (\%) & $76(63.3)$ & 15 & 61 & 0.697 \\
\hline Diabetes Mellitus, n (\%) & $30(25)$ & 6 & 24 & 0.897 \\
\hline MMSE, (Mean/SD) & $20.1(8.4)$ & $20.3(9.2)$ & $20.0(8.2)$ & 0.9 \\
\hline Katz Index, (Mean/SD) & $3.9(2.2)$ & $3.2(2.4)$ & $4.1(2.2)$ & 0.08 \\
\hline
\end{tabular}

Table 1: Characteristics of patients admitted with neck of femur (NOF) fractures: overall patient characteristics comparing those who fell within and after two hours of a meal.

Overall, there was a statistically significant difference $(\mathrm{P}=0.002)$ between the proportion of total falls occurring in the four time categories with the lowest percentage of falls occurring at night, $10.8 \%$ between $0000-0600 \mathrm{~h}$. Over the other time periods of falls, $34.2 \%$ of falls occurred between 0600-1200 h (following breakfast), 30\% between $1200-1800 \mathrm{~h}$ (following lunch), and $25 \%$ occurred between 1800-2400 h (following dinner).

In this study, $20.8 \%$ of NOF fractures occurred within, and $79.2 \%$ after, two hours of meal ingestion. NOF fractures as a result of a fall occurring within two hours of a meal occurred among $34.8 \%$ of patients from residential care and $13.0 \%$ of patients from their own home $(\mathrm{P}=0.009)$. One or more falls in the preceding 12 months occurred among $72 \%$ of patients who sustained a fall within, compared to $49.5 \%$ after, two hours of meal ingestion ( $\mathrm{P}=0.044$. Symptoms of hypotension (giddiness, light-headedness, syncope or blurred vision) prior to the fall were reported more commonly $(24.0 \%)$ by patients who fell within two hours of a meal when compared with those $(6.3 \%)$ who fell after two hours of a meal $(\mathrm{P}=0.032)$. A change in posture prior to a fall was similar between those patients who fell within, and after two hours of a meal $(36 \%$ and $31.6 \%$, respectively $(P=0.903))$.

In the logistic regression model, factors found to be independently associated with NOF fractures occurring within two hours of a meal were being in residential care (OR 3.0, CI 1.009-8.687; $\mathrm{P}=0.048$ ) and experiencing symptoms associated with hypotension prior to the fall (OR 5.2, CI 1.342-20.129; $\mathrm{P}=0.017$ ). There were no significant differences between the two groups for all other variables.

\section{Discussion}

Among older patients admitted with NOF fractures, one-fifth of fractures occurred within two hours of a meal. The majority of NOF fractures occurred during the day with only a small percentage occurring during the night. Also, patients who sustained a NOF fracture within two hours of a meal were more likely to be from residential care, experience symptoms associated with hypotension before a fall and had a history of recurrent falls in the preceding 12 months compared with patients who experienced a fall after two hours of a meal.

The overall distribution of falls across the four time categories was significantly different with the lowest prevalence of falls occurring at night. This is consistent with previous research indicating that injurious falls were more likely to occur during the day and is believed to be related to increased ambulation, which is desirable to maintain physical independence $[25,26]$. It has also previously been reported by Norton et al. [19] that $10.4 \%$ of falls among patients admitted with a fractured femur occurred at night, consistent with our finding.

The significantly higher proportion of older patients from residential care who sustained NOF fractures within two hours of meal ingestion compared to older patients living at home, may be attributed to underlying differences in co-morbidities that affects the risk of falling following a meal. PPH would be one such co-morbidity in view of the accompanying BP changes that occur after a meal. Furthermore, a higher PPH prevalence has been reported among older people in residential care $(24 \%-57 \%)[14,15,27]$ compared to those at home (7\%-30\%) [28].

Patients sustaining a NOF fracture within two hours of a meal were more likely to report symptoms consistent with hypotension 
Page 4 of 5

compared to those falling after two hours of a meal. The absence of a significant difference in change in posture before the fall, between the two groups of patients, may suggest that orthostatic hypotension is less likely to be a contributing factor. It can be speculated that the hypotensive symptoms may be related to $\mathrm{PPH}$. We, therefore, suggest that older people presenting with falls might benefit from being assessed for symptoms of hypotension occurring within two hours of a meal as when $\mathrm{PPH}$ is diagnosed, there are a range of simple nonpharmacological strategies for the management of PPH that could be recommended. For example, drinking water before a meal $[29,30]$ and dietary modification such as consuming smaller portions of meals more frequently and avoiding excessive carbohydrate intake [31]. This may reduce the risk of $\mathrm{PPH}$ and, hence, falls and possible fractures. However, before firm conclusions can be drawn, there is a need to further investigate this theory by undertaking research whereby measurement of blood pressure is undertaken.

A history of recurrent falls in the preceding 12 months, which was significantly more common among older patients who sustained NOF fractures within two hours of a meal, may be attributed to the presence of factors that increase the falls risk in this group. In view that there was no significant difference in the mean Charlson Comorbidity Index between older patients who sustained NOF fractures within and after two hours of a meal, this suggests the presence of co-morbidities that the Charlson Comorbidity Index does not capture.

In considering our observations, it should be recognized that cognitive impairment was present in patients and so there may be inaccuracies pertaining to the actual time of the fall reported in some patients. Furthermore, broad time categories were used to symbolize breakfast, lunch and dinner rather than specifically clarifying when patients had ingested their last meals. This surrogate history measure for PPH was considered more logistically feasible in this population group and was, therefore, employed rather than the more objective evaluation of $\mathrm{BP}$ reduction following a meal. The generalizability of the findings of this study is restricted and future research should consider all patients admitted overnight who have experienced a fall.

In conclusion, in this prospective exploratory study, $20.8 \%$ of NOF fractures occurred within two hours of a meal. Patients who sustained a NOF fracture within two hours of a meal were more likely to be from residential care, experience symptoms associated with hypotension before the fall and had a history of recurrent falls in the preceding 12 months compared with patients who experienced a fall after two hours of a meal. PPH may be a contributing factor in this group of patients, therefore, patients who present with falls and symptoms of hypotension within two hours of a meal could be further investigated for $\mathrm{PPH}$. Where $\mathrm{PPH}$ is diagnosed, simple non-pharmacological strategies may be recommended.

\section{Acknowledgments}

The authors wish to thank Kylie Lange, biostatistician, Centre of Research Excellence in Translating Nutritional Science to Good Health, University of Adelaide, for statistical advice. Data from this manuscript have been presented and awarded the Best Oral Presentation - Clinical Research to Dr Shailaja Nair (Nair S, Gentilcore D, Visvanathan R. 'Is postprandial hypotension a possible contributing factor to hip fractures in older people?') at the Basil Hetzel Institute and The Queen Elizabeth Hospital Research Day, Adelaide, Australia, 2013. The authors have no conflicts of interest to declare in relation to this research.

\section{References}

1. Tinetti ME, Speechley M (1989) Prevention of falls among the elderly.N Engl J Med 320: 1055-1059.

2. Kannus P, Parkkari J, Koskinen S, Niemi S, Palvanen M, et al. (1999) Fallinduced injuries and deaths among older adults. JAMA 281: 1895-1899.

3. Rubenstein LZ, Josephson KR (2002) The epidemiology of falls and syncope. Clin Geriatr Med 18: 141-158

4. Moller J (2003) Projected costs of fall related injury to older persons due to demographic change in Australia. Report to the Commonweath Department of Health and Ageing, under the National Falls Prevention for Older People Initiative. In: Ageing DoHa, ed. Canberra

5. Hannan EL, Magaziner J, Wang JJ, Eastwood EA, Silberzweig SB, et al. (2001) Mortality and locomotion 6 months after hospitalization for hip fracture: risk factors and risk-adjusted hospital outcomes. JAMA 285: 2736-2742.

6. Magaziner J, Simonsick EM, Kashner TM, Hebel JR, Kenzora JE (1990) Predictors of functional recovery one year following hospital discharge for hip fracture: a prospective study. J Gerontol 45: M101-107.

7. Jette AM, Harris BA, Cleary PD, Campion EW (1987) Functional recovery after hip fracture. Arch Phys Med Rehabil 68: 735-740.

8. Johnell O (1997) The socioeconomic burden of fractures: today and in the 21st century. Am J Med 103: 20S-25S.

9. Haentjens P, Lamraski G, Boonen S (2005) Costs and consequences of hip fracture occurrence in old age: an economic perspective. Disabil Rehabil 27: 1129-1141.

10. Auron-Gomez M, Michota F (2008) Medical management of hip fracture. Clin Geriatr Med 24: 701-719, ix.

11. Tinetti ME, Speechley M, Ginter SF (1988) Risk factors for falls among elderly persons living in the community. N Engl J Med 319: 1701-1707.

12. Lipsitz LA (1989) Orthostatic hypotension in the elderly. N Engl J Med 321: 952-957.

13. Rhebergen GA, Schölzel-Dorenbos CJ (2002) [Orthostatic and postprandial hypotension in patients aged 70 years or older admitted to a medical ward]. Tijdschr Gerontol Geriatr 33: 119-123.

14. Aronow WS, Ahn C (1994) Postprandial hypotension in 499 elderly persons in a long-term health care facility. J Am Geriatr Soc 42: 930-932.

15. Le Couteur DG, Fisher AA, Davis MW, McLean AJ (2003) Postprandial systolic blood pressure responses of older people in residential care: association with risk of falling.Gerontology 49: 260-264

16. Aronow WS, Ahn C (1997) Association of postprandial hypotension with incidence of falls, syncope, coronary events, stroke, and total mortality at 29-month follow-up in 499 older nursing home residents.J Am Geriatr Soc 45: 1051-1053.

17. Jansen RW, Connelly CM, Kelley-Gagnon MM, Parker JA, Lipsitz LA (1995) Postprandial hypotension in elderly patients with unexplained syncope.Arch Intern Med 155: 945-952.

18. Mathias CJ, da Costa DF, Fosbraey P, Bannister R, Wood SM, et al. (1989) Cardiovascular, biochemical and hormonal changes during foodinduced hypotension in chronic autonomic failure.J Neurol Sci 94: 255-269.

19. Norton R, Campbell AJ, Lee-Joe T, Robinson E, Butler M (1997) Circumstances of falls resulting in hip fractures among older people.J Am Geriatr Soc 45: 1108-1112.

20. Formiga F, Navarro M, Duaso E, Chivite D, Ruiz D, et al. (2008) Factors associated with hip fracture-related falls among patients with a history of recurrent falling. Bone 43: 941-944.

21. Jansen RW, Lipsitz LA (1995) Postprandial hypotension: epidemiology, pathophysiology, and clinical management. Ann Intern Med 122: 286-295.

22. Charlson ME, Pompei P, Ales KL, MacKenzie CR (1987) A new method of classifying prognostic comorbidity in longitudinal studies: development and validation. J Chronic Dis 40: 373-383. 
Citation: Nair S, Piscitelli D, Tam K, Nair PS, Visvanathan R (2014) Neck of Femur Fractures within Two Hours of a Meal . J Gerontol Geriatr Res 3: 1000194. doi:10.4172/2167-7182.1000194

Page 5 of 5

23. Folstein MF, Folstein SE, McHugh PR (1975) Mini- mental state. A practical method for grading the cognitive state of patients for the clinician. Journal of Psychiatric Research 12: 189-198.

24. Katz S, Ford AB, Moskowitz RW, Jackson BA, Jaffe MW (1963) Studies of Illness in the Aged. The Index of ADL: A Standardized Measure of Biological and Psychosocial Function. JAMA 185: 914-919.

25. Lehtola S, Koistinen P, Luukinen H (2006) Falls and injurious falls late in home-dwelling life. Arch Gerontol Geriatr 42: 217-224.

26. Overstall PW (1992) Falls Review. Clin Gerontol 2: 31-38.

27. Vaitkevicius PV, Esserwein DM, Maynard AK, O'Connor FC, Fleg JL (1991) Frequency and importance of postprandial blood pressure reduction in elderly nursing-home patients.Ann Intern Med 115: 865-870.
28. Lipsitz LA, Fullerton KJ (1986) Postprandial blood pressure reduction in healthy elderly. J Am Geriatr Soc 34: 267-270

29. Deguchi K, Ikeda K, Sasaki I, Shimamura M, Urai Y, et al. (2007) Effects of daily water drinking on orthostatic and postprandial hypotension in patients with multiple system atrophy. J Neurol 254: 735-740.

30. Jones KL, O'Donovan D, Russo A, Meyer JH, Stevens JE, et al. (2005) Effects of drink volume and glucose load on gastric emptying and postprandial blood pressure in healthy older subjects. Am J Physiol Gastrointest Liver Physiol 289: G240-248.

31. Puvi-Rajasingham S, Mathias CJ (1996) Effect of meal size on postprandial blood pressure and on postural hypotension in primary autonomic failure. Clin Auton Res 6: 111-114. 